\title{
ON THE VOLUME OF COMPLEX AMOEBAS
}

\author{
FARID MADANI AND MOUNIR NISSE
}

(Communicated by Lev Borisov)

Dedicated to the memory of Mikael Passare (1959-2011)

\begin{abstract}
The paper deals with amoebas of $k$-dimensional algebraic varieties in the complex algebraic torus of dimension $n \geq 2 k$. First, we show that the area of complex algebraic curve amoebas is finite. Moreover, we give an estimate of this area in the rational curve case in terms of the degree of the rational parametrization coordinates. We also show that the volume of the amoeba of a $k$-dimensional algebraic variety in $\left(\mathbb{C}^{*}\right)^{n}$, with $n \geq 2 k$, is finite.
\end{abstract}

\section{INTRODUCTION}

Amoebas have proven to be a very useful tool in several areas of mathematics, and they have many applications in real algebraic geometry, complex analysis, mirror symmetry, algebraic statistics and in several other areas (see [M1-02, [M2-04, M3-00, FPT-00, NS-11, PR-04, PS-04 and R-01]). They degenerate to piecewise-linear objects called tropical varieties (see [M1-02, [M2-04, and [PR-04]). Moreover, we can use amoebas as an intermediate link between the classical and the tropical geometry.

The amoeba $\mathscr{A}$ of an algebraic variety $V \subset\left(\mathbb{C}^{*}\right)^{n}$ is a closed subset of $\mathbb{R}^{n}$, and its (Lebesgue) volume is well-defined. Passare and Rullgård PR-04 proved that the area of complex plane curve amoebas is finite and the bound is given in terms of the Newton polygon. In this paper, we prove that the amoeba area of any algebraic curve in $\left(\mathbb{C}^{*}\right)^{n}$ is finite (the area here is with respect to the induced Euclidean metric of $\mathbb{R}^{n}$ ). Moreover, we generalize our result, for any algebraic variety $V$ of dimension $k$ in the complex algebraic torus $\left(\mathbb{C}^{*}\right)^{2 k+m}$ with $m \geq 0$. Our main result is the following theorem:

Theorem 1.1. Let $V$ be an algebraic variety of dimension $k$ in the complex torus $\left(\mathbb{C}^{*}\right)^{2 k+m}$ with $m \geq 0$, such that no irreducible component of $V$ is contained in a subtorus of dimension less than $2 k$. Then, the volume of its amoeba is finite.

Note that if $V$ contains an irreducible component in an algebraic subtorus of dimension strictly less than $2 k$, then the volume of its amoeba is infinite.

The remainder of this paper is organized as follows. In Section 2, we review some properties of the amoebas and also the structure theorem of the logarithmic limit set defined by Bergman [B-71], which is used as a tool in the proof of our results.

Received by the editors February 11, 2011 and, in revised form, August 6, 2011.

2010 Mathematics Subject Classification. Primary 14T05, 32A60.

Key words and phrases. Algebraic varieties, amoebas, logarithmic limit sets.

The first author is supported by the Alexander von Humboldt Foundation.

The research of the second author is partially supported by NSF MCS grant DMS-0915245. 
In Section 3, we prove our main result for complex algebraic curves in $\left(\mathbb{C}^{*}\right)^{n}$ for any $n \geq 2$. In Section 4 , we give an estimate of the bound for algebraic rational complex curves and some examples. In Section 5, we prove the main theorem of this paper.

\section{Preliminaries}

Let $V$ be an algebraic variety in $\left(\mathbb{C}^{*}\right)^{n}$. The amoeba $\mathscr{A}$ of $V$ is by definition (see I.M. Gel'fand, M.M. Kapranov and A.V. Zelevinsky [GKZ-94]) the image of $V$ under the map

$$
\begin{aligned}
& \text { Log : } \quad\left(\mathbb{C}^{*}\right)^{n} \quad \longrightarrow \mathbb{R}^{n} \\
& \left(z_{1}, \ldots, z_{n}\right) \longmapsto\left(\log \left|z_{1}\right|, \ldots, \log \left|z_{n}\right|\right) .
\end{aligned}
$$

Passare and Rullgård prove the following (see [PR-04]):

Theorem 2.1. Let $f$ be a Laurent polynomial in two variables. Then the area of the amoeba of the curve with defining polynomial $f$ is not greater than $\pi^{2}$ times the area of the Newton polytope of $f$.

In [MR-00, Mikhalkin and Rullgård show that up to multiplication by a constant in $\left(\mathbb{C}^{*}\right)^{2}$, the algebraic plane curves with Newton polygon $\Delta$ with maximal amoeba area are defined over $\mathbb{R}$. Furthermore, their real loci are isotopic to so-called Harnack curves (possibly singular with ordinary real isolated double points).

Recall that the Hausdorff distance between two closed subsets $A, B$ of a metric space $(E, d)$ is defined by

$$
d_{\mathcal{H}}(A, B)=\max \left\{\sup _{a \in A} d(a, B), \sup _{b \in B} d(A, b)\right\},
$$

where we take $E=\mathbb{R}^{n} \times\left(S^{1}\right)^{n}$, with the distance defined as the product of the Euclidean metric on $\mathbb{R}^{n}$ and the flat metric on $\left(S^{1}\right)^{n}$.

The logarithmic limit set of a complex algebraic variety $V$, denoted by $\mathscr{L}^{\infty}(V)$, is the set of limit points of $\mathscr{A}$ in the sphere $S^{n-1}=\left(\mathbb{R}^{n}\right)^{*} / \mathbb{R}_{+}$. In other words, if $S^{n-1}$ denotes the boundary of the unit ball $B^{n}$ and $r$ the map defined by

$$
\begin{aligned}
r: \quad \mathbb{R}^{n} & \longrightarrow B^{n} \\
x & \longmapsto r(x)=\frac{x}{1+|x|},
\end{aligned}
$$

then $\mathscr{L}^{\infty}(V)=\overline{r(\mathscr{A})} \cap S^{n-1}$. Bergman B-71] proved that if $V \subset\left(\mathbb{C}^{*}\right)^{n}$ is an algebraic variety of dimension $k$, then the cone over $\mathscr{L}^{\infty}(V)$ is contained in a finite union of $k$-dimensional subspaces of $\mathbb{R}^{n}$ defined over $\mathbb{Q}$. On the other hand, Bieri and Groves BG-84 proved that this cone is a finite union of rational polyhedron convex cones of dimension at most $k$, and the maximal dimension in this union is achieved by at least one polyhedron $P$ in this union. Moreover, one has $\operatorname{dim}_{\mathbb{R}} \mathscr{L}^{\infty}(V)=\operatorname{dim}_{\mathbb{C}} V-1$. More precisely, we have the following structure theorem:

Theorem 2.2 (Bergman, Bieri-Groves). The logarithmic limit set $\mathscr{L}^{\infty}(V)$ of an algebraic variety $V$ in $\left(\mathbb{C}^{*}\right)^{n}$ is a finite union of rational spherical polyhedrons. The maximal dimension of a polyhedron in this union is achieved at least by one polyhedron $P$ in this union, and we have $\operatorname{dim}_{\mathbb{R}} \mathscr{L}^{\infty}(V)=\operatorname{dim}_{\mathbb{R}} P=\operatorname{dim}_{\mathbb{C}} V-1$. 


\section{Area of complex algebraic Curve amoebas}

The main result of this section is the following theorem:

Theorem 3.1. Let $\mathcal{C} \subset\left(\mathbb{C}^{*}\right)^{n}$ be an algebraic curve with $n \geq 2$. Assume that no irreducible component of $\mathcal{C}$ is contained in a subtorus of dimension less than 2. Then, the area of its amoeba is finite.

We start by proving Theorem 3.1 in the rational curve case (see Theorem 3.2). Recall that a complex algebraic curve is contained in a subtorus of dimension one, meaning that the curve is the subtorus of dimension one itself (sometimes called a holomorphic annulus). Moreover, its amoeba is a straight line in $\mathbb{R}^{n}$, and this case is not interesting for us because it is not generic (i.e., the Jacobian matrix of the logarithmic map is not of maximal rank).

Let $n$ and $k$ be two positive integers such that $2 k \leq n$. Let $f: \mathbb{C}^{k} \longrightarrow\left(\mathbb{C}^{*}\right)^{n}$ be a rational map, and $V$ be the variety in $\left(\mathbb{C}^{*}\right)^{n}$ defined by the image of $f$. We denote by $\left\{z_{j}\right\}_{1 \leq j \leq k}$ the complex coordinates of $\mathbb{C}^{k}$, and by $\left\{f_{j}\right\}_{1 \leq j \leq n}$ the coordinates of $f$ in $\mathbb{C}^{n}$. For simplicity, we denote by $\log f$ the composition $\log \circ f$.

Let $\mathscr{A}_{f}$ be the amoeba of $V$ (i.e. $\mathscr{A}_{f}=\log (V)$ ). Let $S$ be the set of points in $\mathbb{C}^{k}$ defined by

$$
S=\left\{z \in \mathbb{C}^{k} \mid \operatorname{rank}_{z} \log f<2 k\right\}
$$

and $\mathscr{S}_{f}=\log f(S)$ be the set of critical values of $\log f$.

By construction, $\log f$ is an immersion from $\mathbb{C}^{k} \backslash S$ to $\mathbb{R}^{n}$. Hence, the set $\mathscr{A}_{f} \backslash \mathscr{S}_{f}=\log f\left(\mathbb{C}^{k} \backslash S\right)$ is a real $2 k$-dimensional immersed submanifold in $\mathbb{R}^{n}$. We endow $\mathscr{A}_{f} \backslash \mathscr{S}_{f}$ with the induced Riemannian metric $\imath^{*} \mathcal{E}_{n}$, where $\mathcal{E}_{n}$ is the Euclidean metric of $\mathbb{R}^{n}$ and $\imath: \mathscr{A}_{f} \backslash \mathscr{S}_{f} \hookrightarrow \mathbb{R}^{n}$ is the inclusion map. Let $U_{f} \subset \mathbb{C}^{k} \backslash S$ be an open set such that $\left.\log f\right|_{U_{f}}$ is an injective immersion and $\log f\left(U_{f}\right)=\mathscr{A}_{f} \backslash \mathscr{S}_{f}$. We claim that

$$
\operatorname{vol}\left(\mathscr{A}_{f} \backslash \mathscr{S}_{f}, \imath^{*} \mathcal{E}_{n}\right)=\operatorname{vol}\left(U_{f},(\log f)^{*} \mathcal{E}_{n}\right),
$$

where $\operatorname{vol}\left(\mathscr{A}_{f} \backslash \mathscr{S}_{f}, \imath^{*} \mathcal{E}_{n}\right)$ is the volume of $\mathscr{A}_{f} \backslash \mathscr{S}_{f}$ with respect to the metric $\imath^{*} \mathcal{E}_{n}$. Let $\psi_{2 k}$ be a real $2 k$-vector field in $\Lambda^{2 k} \mathbb{C}^{k}$ which does not vanish on $\mathbb{C}^{k}$ and $\mathrm{d} v_{(\log f)^{*} \mathcal{E}_{n}}, \mathrm{~d} v_{\mathcal{E}_{2 k}}$ be the volume forms defined over $U_{f}$ associated to the metrics $(\log f)^{*} \mathcal{E}_{n}$ and $\mathcal{E}_{2 k}$ respectively. These two forms are related by the following formula:

$$
\left|\psi_{2 k}\right|_{\mathcal{E}_{2 k}} \mathrm{~d} v_{(\log f)^{*} \mathcal{E}_{n}}=\left|\psi_{2 k}\right|_{(\log f)^{*} \mathcal{E}_{n}} \mathrm{~d} v_{\mathcal{E}_{2 k}} .
$$

Now we choose $\psi_{2 k}$ such that $\mathrm{d} v_{\mathcal{E}_{2 k}}\left(\psi_{2 k}\right)=\left|\psi_{2 k}\right|_{\mathcal{E}_{2 k}}=1$. From (11) and (2) we deduce that

$$
\operatorname{vol}\left(\mathscr{A}_{f} \backslash \mathscr{S}_{f}\right)=\int_{U_{f}}\left|\psi_{2 k}\right|_{(\log f) * \mathcal{E}_{n}} \mathrm{~d} v_{\mathcal{E}_{2 k}},
$$

and area $:=v o l$ if $k=1$. This definition of the volume does not depend on the choice of coordinates. It is more convenient to use the following integral vol $_{2 k}$ defined as

$$
\operatorname{vol}_{2 k}\left(\mathscr{A}_{f} \backslash \mathscr{S}_{f}\right)=\int_{\mathbb{C}^{k}-S}\left|\psi_{2 k}\right|_{(\log f)^{*} \mathcal{E}_{n}} \mathrm{~d} v_{\mathcal{E}_{2 k}}
$$


Remark 3.1. $\operatorname{vol}_{2 k}\left(\mathscr{A}_{f} \backslash \mathscr{S}_{f}\right)$ can be viewed as a weighted volume of $\mathscr{A}_{f} \backslash \mathscr{S}_{f}$. In fact, we know that there exist a positive integer $m$ and a family of open connected components on $\mathscr{A}_{f} \backslash \mathscr{S}_{f}$, denoted by $\left\{R_{\alpha}\right\}$, such that

$$
\mathscr{A}_{f} \backslash \mathscr{S}_{f}=\bigsqcup_{\alpha=1}^{m} R_{\alpha}, \quad(\log f)^{-1} R_{\alpha}=\bigsqcup_{\beta=1}^{p_{\alpha}} U_{\alpha \beta}, \quad \bigsqcup_{\alpha=1}^{m} \bigsqcup_{\beta=1}^{p_{\alpha}} U_{\alpha \beta}=\mathbb{C}^{k} \backslash S
$$

and the map $\log f: U_{\alpha \beta} \longrightarrow R_{\alpha}$ is a diffeomorphism, for all $\alpha \leq m$ and $1 \leq \beta \leq p_{\alpha}$.

For a chosen $\beta$, we set $U_{f}=\bigsqcup_{\alpha=1}^{m} U_{\alpha \beta}$. This yields

$$
\begin{aligned}
\operatorname{vol}_{2 k}\left(\mathscr{A}_{f} \backslash \mathscr{S}_{f}\right) & =\sum_{\alpha=1}^{m} p_{\alpha} \int_{U_{\alpha \beta}}\left|\psi_{2 k}\right|_{(\log f) * \mathcal{E}_{n}} \mathrm{~d} v_{\mathcal{E}_{2 k}}, \\
\operatorname{vol}\left(\mathscr{A}_{f} \backslash \mathscr{S}_{f}\right) & =\sum_{\alpha=1}^{m} \int_{U_{\alpha \beta}}\left|\psi_{2 k}\right|_{(\log f)^{*} \mathcal{E}_{n}} \mathrm{~d} v_{\mathcal{E}_{2 k}} .
\end{aligned}
$$

If we define $p=\min _{1 \leq \alpha \leq m} p_{\alpha}$ and $P=\max _{1 \leq \alpha \leq m} p_{\alpha}$, then

$$
\frac{\operatorname{vol}_{2 k}\left(\mathscr{A}_{f} \backslash \mathscr{S}_{f}\right)}{P} \leq \operatorname{vol}\left(\mathscr{A}_{f} \backslash \mathscr{S}_{f}\right) \leq \frac{\operatorname{vol}_{2 k}\left(\mathscr{A}_{f} \backslash \mathscr{S}_{f}\right)}{p}
$$

On $\mathbb{C}^{k}, \mathrm{~d} v_{\mathcal{E}_{2 k}}$ and $\psi_{2 k}$ are given by

$$
\mathrm{d} v_{\mathcal{E}_{2 k}}=i^{k} \mathrm{~d} z \wedge \mathrm{d} \bar{z}, \quad \psi_{2 k}=(-i)^{k} \frac{\partial}{\partial z} \wedge \frac{\partial}{\partial \bar{z}},
$$

where $\mathrm{d} z=\mathrm{d} z_{1} \wedge \cdots \wedge \mathrm{d} z_{k}$ and $\frac{\partial}{\partial z}=\frac{\partial}{\partial z_{1}} \wedge \cdots \wedge \frac{\partial}{\partial z_{k}}$.

Let us now compute $\left|\psi_{2 k}\right|_{(\log f)^{*} \mathcal{E}_{n}}$. We have

$$
\frac{\partial \log f}{\partial z} \wedge \frac{\partial \log f}{\partial \bar{z}}=\sum_{I=\left\{i_{1}<\cdots<i_{2 k}\right\}} \operatorname{det}\left(\partial_{j} \log f_{I}\right)_{1 \leq j \leq 2 k} e_{I},
$$

where for all $I=\left\{i_{1}<\cdots<i_{2 k}\right\} \subset\{1, \ldots, n\}, f_{I}=\left(f_{i_{1}}, \ldots, f_{i_{2 k}}\right),\left\{e_{j}\right\}_{1 \leq j \leq n}$ is an orthonormal basis of $T^{*} \mathbb{R}^{n}$ and $e_{I}:=e_{i_{1}} \wedge \cdots \wedge e_{i_{2 k}}$. This implies that

$$
\left\{e_{I}\right\}_{I \subset\{1, \ldots, n\},|I|=2 k}
$$

is an orthonormal basis of $\wedge^{2 k} T^{*} \mathbb{R}^{n}$ with respect to the Euclidean metric. We denote by $\partial_{j}=\frac{\partial}{\partial z_{j}}$ if $j \leq k$ and by $\partial_{j}=\frac{\partial}{\partial \bar{z}_{j-k}}$ if $j \geq k+1$. Then

$$
\left|\psi_{2 k}\right|_{(\log f)^{*} \mathcal{E}_{n}}^{2}=\sum_{I=\left\{i_{1}<\cdots<i_{2 k}\right\}}\left|\operatorname{det}\left(\partial_{j} \log f_{I}\right)_{1 \leq j \leq 2 k}\right|^{2} .
$$

Hence, we deduce the following inequality:

$$
\left|\psi_{2 k}\right|_{(\log f)^{*} \mathcal{E}_{n}} \leq \sum_{I=\left\{i_{1}<\cdots<i_{2 k}\right\}}\left|\operatorname{det}\left(\partial_{j} \log f_{I}\right)_{1 \leq j \leq 2 k}\right| .
$$

We have the following result:

Theorem 3.2. Let $f: \mathbb{C} \longrightarrow\left(\mathbb{C}^{*}\right)^{n}$ be a rational map, and $\mathcal{C}$ be the rational curve of $\left(\mathbb{C}^{*}\right)^{n}$ defined by the image of $f$. The area of $\mathscr{A}_{f}$ with respect to the Euclidean metric of $\mathbb{R}^{n}$ is finite.

Lemma 3.1. Let $f=\left(f_{1}, f_{2}\right)$ be a rational map from $\mathbb{C}$ to $\mathbb{C}^{2}$. The function $i \operatorname{det}\left(\partial_{z} \log f, \partial_{\bar{z}} \log f\right)$ is a real-valued rational function. Moreover,

(i) The map $f$ has simple poles. 
(ii) There exist $P, Q \in \mathbb{R}[X, Y]$ such that $i \operatorname{det}\left(\partial_{z} \log f, \partial_{\bar{z}} \log f\right)=\frac{P}{Q}$ with $\operatorname{deg} Q \geq \operatorname{deg} P+3$.

Proof. We have

$$
i \operatorname{det}\left(\partial_{z} \log f, \partial_{\bar{z}} \log f\right)=\frac{i}{4}\left(\frac{f_{1}^{\prime}}{f_{1}} \frac{\bar{f}_{2}^{\prime}}{\bar{f}_{2}}-\frac{\bar{f}_{1}^{\prime}}{\bar{f}_{1}} \frac{f_{2}^{\prime}}{f_{2}}\right) .
$$

It is trivial that this function, which is $i$ times the Jacobian determinant of $\log f$, is a real rational function. Its poles are the zeros and the poles of $f$ and their order is equal to one (even if there is a common pole or zero of $f_{1}$ and $f_{2}$, one can check that this pole is also simple). On the other hand, if $i \operatorname{det}\left(\partial_{z} \log f, \partial_{\bar{z}} \log f\right)=\frac{P}{Q}$, then $\operatorname{deg} Q \geq \operatorname{deg} P+2$. This inequality can be improved. In fact, by elementary computations on the $\operatorname{deg} P$ and $\operatorname{deg} Q$, we show that $\operatorname{deg} Q \geq \operatorname{deg} P+3$.

Proof of Theorem 3.2. First of all, we do not have to worry about the area of $\mathscr{S}_{f}$. Indeed, by Sard's theorem, we know that this area is equal to zero. If $\mathscr{A}_{f} \backslash \mathscr{S}_{f}$ is empty, then the area of $\mathscr{A}_{f}$ is zero. From now on, we assume that $\mathscr{A}_{f} \backslash \mathscr{S}_{f}$ is not empty. Hence, it is a surface defined in $\mathbb{R}^{n}$ and $\mathscr{A}_{f} \backslash \mathscr{S}_{f}, \mathscr{A}_{f}$ have the same area.

The area of $\mathscr{A}_{f} \backslash \mathscr{S}_{f}$ is given by (3). Hence, it is sufficient to prove that $\left|\psi_{2}\right|$ is integrable over $\mathbb{C}$. Inequality (9) implies that

$$
\left|\psi_{2}\right|_{(\log f)^{*} \mathcal{E}_{n}} \leq \sum_{1 \leq j<k \leq n}\left|\operatorname{det}\left(\partial_{z} \log f_{j}, \partial_{\bar{z}} \log f_{k}\right)\right| .
$$

We claim that all the functions on the right-hand side of (10) are integrable. Indeed, using Lemma 3.1. $(i)$, we have the integrability in a neighborhood of any pole. By $(i i)$, we get the integrability at infinity.

Proof of Theorem 3.1. Let $\mathcal{C}$ be an algebraic curve in $\left(\mathbb{C}^{*}\right)^{n}$. Then its closure $\overline{\mathcal{C}}$ in $\mathbb{C P}^{n}$ is an algebraic curve. Hence, any end of $\mathcal{C}$ corresponds to a local branch of $\overline{\mathcal{C}}$ at some point $p \in \partial \overline{\mathcal{C}} \backslash \mathcal{C}$. After a monomial map of $\left(\mathbb{C}^{*}\right)^{n}$ if necessary, we may assume that $p$ corresponding to an end of $\mathcal{C}$ is the origin of $\mathbb{C}^{n}$ in $\mathbb{C P}^{n}$.

A local parametrization $\rho_{p}$ of a branch of $\overline{\mathcal{C}}$ at $p$ can be written in terms of vectorial Puiseux series in $t$ near zero as follows:

$$
\begin{aligned}
\rho_{p}: \quad \mathbb{C}^{*} & \longrightarrow\left(\mathbb{C}^{*}\right)^{n} \\
t & \longmapsto\left(b_{1} t^{u_{1}}, \ldots, b_{n} t^{u_{n}}\right),
\end{aligned}
$$

where $\left(b_{1}, \ldots, b_{n}\right) \in\left(\mathbb{C}^{*}\right)^{n}$, and $\left(u_{1}, \ldots, u_{n}\right) \in \mathbb{Q}_{\geq 0}^{n}$. Indeed, the Bergman logarithmic limit set of a curve is a finite number of points $\left\{v_{i}\right\}$ on the sphere $S^{n-1}$ (see [B-71]). By Bieri and Groves (see BG-84]), if $O$ denotes the origin of $\mathbb{R}^{n}$, then the slope $\vec{u}$ of the real line $\left(O v_{i}\right)$ in $\mathbb{R}^{n}$ is rational (the slope here means the direction vector of the line, and rational means that its coordinates are rational). Hence, there exist real lines $L_{\vec{u}, j}$ in $\mathbb{R}^{n}$ parametrized by

$$
x \longmapsto\left(a_{1}^{j}+x u_{1}, \ldots, a_{n}^{j}+x u_{n}\right)
$$

with $x \in \mathbb{R}$ and $\left(a_{1}^{j}, \ldots, a_{n}^{j}\right) \in \mathbb{R}^{n}$, such that the amoebas $\mathscr{A}_{h}$ of $h$ in the defining ideal of the curve $\mathcal{C}$ reach all these lines at the infinity in the direction $\vec{u}$. So, for each line $L_{\vec{u}, j}$ there exists $\left(b_{1}, \ldots, b_{n}\right) \in\left(\mathbb{C}^{*}\right)^{n}$ such that the Hausdorff distance between the complex line $\mathcal{L}_{\vec{u}, j}$ parametrized by:

$$
t \longmapsto\left(b_{1} t^{u_{1}}, \ldots, b_{n} t^{u_{n}}\right),
$$


and $V_{h} \cap \log ^{-1}\left(L_{\vec{u}, j} \backslash B(O, R)\right)^{+}$tends to zero when $R$ goes to infinity, where $\left(L_{\vec{u}, j} \backslash B(O, R)\right)^{+}$denotes the component which is in the direction of $\vec{u}$. Hence, the Hausdorff distance between the intersection of the curve with $\log ^{-1}\left(L_{\vec{u}, j} \backslash\right.$ $B(O, R))^{+}$and $\mathcal{L}_{\vec{u}, j}$ tends to zero for $R$ sufficiently large. Now, using Theorem 3.2 and the fact that the number of ends of an algebraic curve is finite, we obtain the result.

\section{An estimate for the area of Rational Curve amoebas}

In this section, we assume that $k=1$. Recall that $f$ is the rational map defined in Section 3 with $k=1$. Hence, for any integer $j \in[1, n]$ :

$$
f_{j}(z)=c \prod_{\ell=1}^{d_{j}}\left(z-a_{j \ell}\right)^{m_{j \ell}}
$$

where $a_{j \ell}$ are distinct poles or zeros of $f_{j}, m_{j \ell}$ are their multiplicities $\left(m_{j \ell}\right.$ are negative in the case of poles) and $d_{j}$ is the number of distinct zeros and poles of $f_{j}$. We define the positive integers $n_{j}:=\sum_{\ell=1}^{d_{j}}\left|m_{j \ell}\right|$. These integers represent the number of poles and zeros of $f_{j}$ counted with their multiplicities.

Theorem 4.1. Let $p$ be the positive integer defined by

$$
p=\min _{x \in \mathscr{A}_{f} \backslash \mathscr{S}_{f}} \#(\log f)^{-1} x .
$$

The following inequalities always hold:

$$
p \cdot \operatorname{area}\left(\mathscr{A}_{f}\right) \leq \operatorname{vol}_{2}\left(\mathscr{A}_{f}\right) \leq \pi^{2} \sum_{1 \leq j_{1}<j_{2} \leq n} n_{j_{1}} n_{j_{2}} .
$$

Moreover, $p \cdot \operatorname{area}\left(\mathscr{A}_{f}\right)=\operatorname{vol}_{2}\left(\mathscr{A}_{f}\right)$ if and only if $\log f: \mathbb{C}^{k} \backslash S \longrightarrow \mathscr{A}_{f} \backslash \mathscr{S}_{f}$ is a covering with exactly $p$ sheets.

Proof. Recall that $v l_{2}$ is defined by (4).

If $n=2$, using (4), (8), we obtain

$$
\operatorname{vol}_{2}\left(\mathscr{A}_{f}\right) \leq \sum_{\ell=1, \ell^{\prime}=1}^{d_{1}, d_{2}}\left|m_{1 \ell} m_{2 \ell^{\prime}}\right| \operatorname{vol}_{2}\left(\mathscr{A}_{f_{a_{1 \ell}, a_{2 \ell^{\prime}}}}\right),
$$

with $f_{a_{1 \ell}, a_{2 \ell^{\prime}}}(z)=\left(z-a_{1 \ell}, z-a_{2 \ell}\right)$. We know that $\operatorname{vol}_{2}\left(\mathscr{A}_{f_{a_{1 \ell}, a_{2 \ell^{\prime}}}}\right)=\pi^{2}$ (it can be proven, using the substitution $z=\left(a_{1 \ell}-a_{2 \ell}\right) t+a_{1 \ell}$ and Example 1 below for $m=1$, which is a plane line). Hence,

$$
\operatorname{vol}_{2}\left(\mathscr{A}_{f}\right) \leq \pi^{2} \sum_{\ell=1, \ell^{\prime}=1}^{d_{1}, d_{2}}\left|m_{1 \ell} m_{2 \ell^{\prime}}\right| .
$$

If $n \geq 2$, using (9) and integrating, we obtain

$$
\operatorname{vol}_{2}\left(\mathscr{A}_{f}\right) \leq \sum_{1 \leq j_{1}<j_{2} \leq n} \operatorname{vol}_{2}\left(\mathscr{A}_{\left(f_{j_{1}}, f_{j_{2}}\right)}\right) .
$$

A combination of (12) and (13) yields

$$
\operatorname{vol}_{2}\left(\mathscr{A}_{f}\right) \leq \pi^{2} \sum_{1 \leq j_{1}<j_{2} \leq n} \sum_{\ell=1, \ell^{\prime}=1}^{d_{j_{1}}, d_{j_{2}}}\left|m_{j_{1} \ell} m_{j_{2} \ell^{\prime}}\right| .
$$


Hence

$$
\operatorname{vol}_{2}\left(\mathscr{A}_{f}\right) \leq \pi^{2} \sum_{1 \leq j_{1}<j_{2} \leq n} n_{j_{1}} n_{j_{2}},
$$

which gives the second inequality of Theorem 4.1 The first one is a consequence of (7).

Assume that $p \cdot \operatorname{area}\left(\mathscr{A}_{f}\right)=\operatorname{vol}_{2}\left(\mathscr{A}_{f}\right)$. Using (5), (6) , we obtain that $p_{\alpha}=p$, for all $\alpha \leq m$. This means that the number of connected components in $(\log f)^{-1} R_{\alpha}$ does not depend on $\alpha$ and $\log f$ is a $p$-sheet covering map.

If we suppose that $\log f: \mathbb{C}^{k} \backslash S \longrightarrow \mathscr{A}_{f} \backslash \mathscr{S}_{f}$ is a covering with exactly $p$ sheets, then (17) becomes an equality.

\section{Examples.}

(1) Let us compute the area of the amoeba $\mathscr{A}_{f}$ with $f(z)=\left(z, z^{m}-1\right)$ and $m \in \mathbb{Z}^{*}$. The set of singular points $S$ is the union of the half lines given by

$$
S=\bigcup_{j=1}^{2|m|}\left\{z \in \mathbb{C} \mid \arg z=\frac{j \pi}{|m|}\right\} .
$$

The set of critical values is $\mathscr{S}_{f}=\log f(S)$, which is a curve in $\mathbb{R}^{2}$ and bounds $\mathscr{A}_{f}$. The map $\log f$ is a covering map with exactly $2|m|$ sheets and for any integer $j \in[1,2|m|]$ we have that

$$
\log f: U_{j}=\left\{z \in \mathbb{C}^{*} \mid \frac{(j-1) \pi}{|m|}<\arg z<\frac{j \pi}{|m|}\right\} \longrightarrow \mathscr{A}_{f}-\mathscr{S}_{f}
$$

is a diffeomorphism. Hence, $\log f$ is a $2|m|-$ sheets covering map. By (프) and (8) we obtain

$\operatorname{area}\left(\mathscr{A}_{f}\right)=\int_{U_{1}}\left|\operatorname{det}\left(\frac{\partial \log f}{\partial z}, \frac{\partial \log f}{\partial \bar{z}}\right)\right| i \mathrm{~d} z \wedge \mathrm{d} \bar{z}=\int_{U_{1}} \frac{|m|\left|z^{m}-\bar{z}^{m}\right|}{4|z|^{2}\left|z^{m}-1\right|^{2}} i \mathrm{~d} z \wedge \mathrm{d} \bar{z}=\frac{\pi^{2}}{2|m|}$, with area $=$ vol. We deduce that $\operatorname{vol}_{2}\left(\mathscr{A}_{f}\right)=2|m| \operatorname{area}\left(\mathscr{A}_{f}\right)=\pi^{2}$.

(2) Now, let us consider the real line in $\left(\mathbb{C}^{*}\right)^{3}$ parametrized by $g(z)=(z$, $\left.z+\frac{1}{2}, z-\frac{3}{2}\right)$. The amoeba $\mathscr{A}_{g}$ is a surface in $\mathbb{R}^{3}$ with boundary as we can see in Figure 1 (this fact is proven in [NP-10). The set of singular points is the line of real points, and $\log g$ is a 2 -sheets covering map. It is complicated to compute the area of $\mathscr{A}_{g}$. However, using the estimate given in Theorem 4.1, we deduce that 2 area $\left(\mathscr{A}_{g}\right)=\operatorname{vol}_{2}\left(\mathscr{A}_{g}\right) \leq 3 \pi^{2}$.

(3) Let $h(z)=(z, z+1, z-2 i)$ be the parametrization of a complex line in $\left(\mathbb{C}^{*}\right)^{3}$. The amoeba $\mathscr{A}_{h}$ is a surface in $\mathbb{R}^{3}$ without boundary as we can see in Figure 2, and topologically it is a Riemann sphere with four marked points. Note that this line is not real and the set of critical values of $\mathrm{Log}$ restricted to this line is empty. The map $\log h: \mathbb{C}-\{-1,0,2 i\} \longrightarrow \mathscr{A}_{h}$ is a diffeomorphism. By Theorem 4.1, area $\left(\mathscr{A}_{h}\right)=\operatorname{vol}_{2}\left(\mathscr{A}_{h}\right) \leq 3 \pi^{2}$.

Passare and Rullgård give an estimate for amoeba areas of complex algebraic plane curves (see Theorem 2.1). Our estimate works only for rational curves immersed in $\left(\mathbb{C}^{*}\right)^{n}$. However, in this case we have a finer estimate (see Theorem 4.1). Indeed, if we consider Example 1, $\operatorname{area}\left(\mathscr{A}_{f}\right)=\frac{\pi^{2}}{2 m}, n_{1}=1, n_{2}=m, p=2 m$ and the area of Newton's polygon is $\frac{m}{2}$. Inequality (11) gives area $\left(\mathscr{A}_{f}\right) \leq \frac{\pi^{2}}{2}$, and the Passare-Rullgård estimate gives $\operatorname{area}\left(\mathscr{A}_{f}\right) \leq \frac{m \pi^{2}}{2}$. 


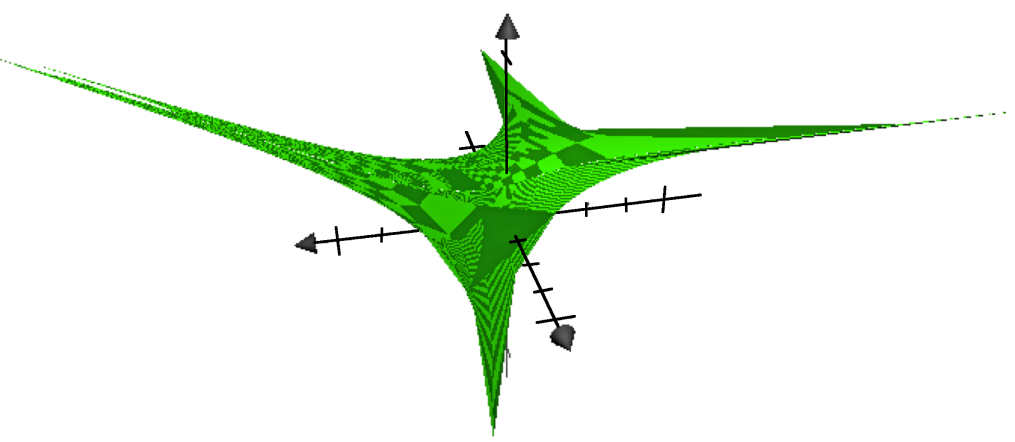

Figure 1. The amoeba of the real line in $\left(\mathbb{C}^{*}\right)^{3}$ given by the parametrization $g(z)=\left(z, z+\frac{1}{2}, z-\frac{3}{2}\right)$.

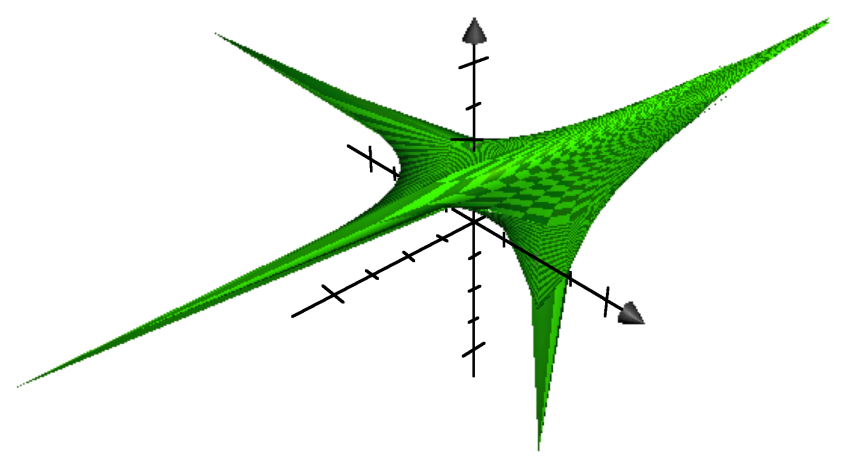

Figure 2. The complex line in $\left(\mathbb{C}^{*}\right)^{3}$ given by the parametrization $h(z)=(z, z+1, z-2 i)$. 


\section{Volume OF GENERIC COMPleX Algebraic VARIETy AMOEBAS}

In this section, we assume that $n=2 k+m$ is an integer with $k \geq 1$ and $m \geq 0$. Let $V \subset\left(\mathbb{C}^{*}\right)^{n}$ be an algebraic variety of dimension $k$ with defining ideal $\mathcal{I}(V)$ and let $\mathscr{L}^{\infty}(V)$ be its logarithmic limit set. We denote by $\operatorname{Vert}\left(\mathscr{L}^{\infty}(V)\right)$ the set of vertices of $\mathscr{L}^{\infty}(V)$. Let $V \subset\left(\mathbb{C}^{*}\right)^{n}$ be a generic algebraic variety of dimension $k$. Let $v \in \operatorname{Vert}\left(\mathscr{L}^{\infty}(V)\right)$ and let us denote by $\mathscr{H}_{R, v}$ the hyperplane in $\mathbb{R}^{n}$ with normal the vector $\overrightarrow{O v}$ such that $d\left(O, \mathscr{H}_{R, v}\right)=R$, where $O$ is the origin of $\mathbb{R}^{n}$ and $R \in \mathbb{R}_{+}$is sufficiently large. We denote by $\mathscr{H}_{R, v}^{-}$the half space with boundary $\mathscr{H}_{R, v}$ containing the origin.

In this section, we prove Theorem 1.1, using the following proposition:

Proposition 5.1. With the above notation, $V \backslash \log ^{-1}\left(\mathscr{H}_{R, v}^{-}\right)$is a fibration over an algebraic variety $V_{v}$ of dimension $k-1$ contained in $\left(\mathbb{C}^{*}\right)^{n-1}$, and its fibers are the ends of algebraic curves in $\left(\mathbb{C}^{*}\right)^{n}$. Moreover, these ends have a rational parametrization with the same slope (i.e., their image under $\log$ are lines with the same slope).

We start by proving the following lemma:

Lemma 5.1. Let $V \subset\left(\mathbb{C}^{*}\right)^{n}$ be an algebraic variety of dimension $k$. Then, for each vertex $v$ of its logarithmic limit set $\mathscr{L}^{\infty}(V)$, we have the following: there exists a complex algebraic variety $V_{v} \subset\left(\mathbb{C}^{*}\right)^{n-1}$ of dimension $k-1$ such that the boundary of the Zariski closure $\bar{V}$ of $V$ in $\left(\mathbb{C}^{*}\right)^{n-1} \times \mathbb{C}$ is equal to $V_{v}$ (i.e., $\partial \bar{V}=\bar{V} \backslash V=V_{v}$, where $\left.\left(\mathbb{C}^{*}\right)^{n-1}=\left(\mathbb{C}^{*}\right)^{n-1} \times\{0\} \subset\left(\mathbb{C}^{*}\right)^{n-1} \times \mathbb{C}\right)$.

Proof. If $v$ belongs to $\operatorname{Vert}\left(\mathscr{L}^{\infty}(V)\right)$, then after a monomial map defined by a ma$\operatorname{trix} A_{v} \in G L_{n}(\mathbb{Z})$ if necessary, we may assume that $v=(0, \ldots, 0,-1) \in \mathbb{S}^{n-1}$. Let $\mathbb{C}\left[z_{1}^{ \pm 1}, \ldots, z_{n-1}^{ \pm 1}, z_{n}\right] \subset \mathbb{C}\left[z_{1}^{ \pm 1}, \ldots, z_{n}^{ \pm 1}\right]$ be the inclusion of rings and $\phi: \mathbb{C}\left[z_{1}^{ \pm 1}, \ldots\right.$, $\left.z_{n-1}^{ \pm 1}, z_{n}\right] \rightarrow \mathbb{C}\left[z_{1}^{ \pm 1}, \ldots, z_{n-1}^{ \pm 1}\right]$ be the homomorphism which sends $z_{n}$ to zero. Let $\mathcal{J}=\mathcal{I}(V) \cap \mathbb{C}\left[z_{1}^{ \pm 1}, \ldots, z_{n-1}^{ \pm 1}, z_{n}\right]$ and $\mathcal{I}_{v}$ be its image in $\mathbb{C}\left[z_{1}^{ \pm 1}, \ldots, z_{n-1}^{ \pm 1}\right]$. We denote by $V_{(n-1)}$ the subvariety of $\left(\mathbb{C}^{*}\right)^{n-1} \times \mathbb{C}$ defined by $\mathcal{J}$, and by $V_{v}=$ $V_{(n-1)} \cap\left(\mathbb{C}^{*}\right)^{n-1} \times\{0\}$ the subvariety defined by $\mathcal{I}_{v}$. We check that $V_{(n-1)}=\bar{V}$ where $\bar{V}$ denotes the Zariski closure of $V$ in $\left(\mathbb{C}^{*}\right)^{n-1} \times \mathbb{C}$ and $V_{v}$ is the boundary of $\bar{V}$ i.e., $\partial \bar{V}=\bar{V} \backslash V=V_{v}$.

Proof of Proposition 5.1. For each point $x$ in $V_{v}$ there exists an algebraic curve $\mathcal{C}_{x}$ in $V$ such that its closure in $\left(\mathbb{C}^{*}\right)^{n-1} \times \mathbb{C}$ contains $x$ and its logarithmic limit set contains the point $v$. Indeed, we have the following commutative diagram:

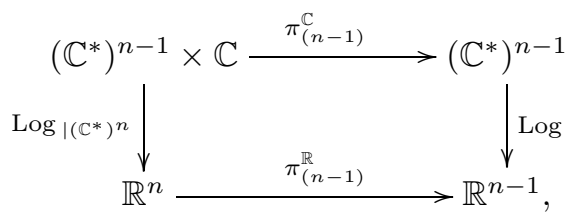

where $\pi_{(n-1)}^{\mathbb{C}}$ and $\pi_{(n-1)}^{\mathbb{R}}$ are the projections on $\left(\mathbb{C}^{*}\right)^{n-1}$ and $\mathbb{R}^{n-1}$ respectively. The limit of $\pi_{(n-1)}^{\mathbb{C}}\left(V \backslash \mathscr{H}_{v, R}^{-}\right)$when $R$ goes to infinity is equal to $V_{v}$ (with respect to the Hausdorff metric on compact sets). Furthermore, the limit of $\pi_{(n-1)}^{\mathbb{R}}(\log (V \backslash$ $\left.\mathscr{H}_{v, R}^{-}\right)$) when $R$ goes to infinity is equal to the amoeba of $V_{v}$. Hence, the limit of 
$\pi_{(n-1)}^{\mathbb{C}}\left(\mathcal{C}_{x} \backslash \mathscr{H}_{v, R}^{-}\right)$when $R$ goes to infinity contains the point $x$. The end of $\mathcal{C}_{x}$ corresponding to $v$ and containing $x$ is parametrized as

$$
\begin{aligned}
\rho_{v}: \quad \mathbb{C}^{*} & \longrightarrow\left(\mathbb{C}^{*}\right)^{n} \\
t & \longmapsto\left(b_{x, 1} t^{u_{1}}, \ldots, b_{x, n} t^{u_{n}}\right),
\end{aligned}
$$

where the coefficients $b_{x, j}$ depend on the holomorphic branch of $\overline{\mathcal{C}_{x}}$ at $x$, and the powers $u_{j}$ depend only on $v$. Moreover, for any $x_{1} \neq x_{2}$ in $V_{v}$ the end of the curve $\mathcal{C}_{x_{1}}$ corresponding to $x_{1}$, and the end of the curve $\mathcal{C}_{x_{2}}$ corresponding to $x_{2}$ have an empty intersection. It may be that the curves $\mathcal{C}_{x_{1}}$ and $\mathcal{C}_{x_{2}}$ are the same. In fact, in this case this means that the curve has more than one end corresponding to $v$. In other words, if $\overline{\mathcal{C}}_{x_{1}}$ is the Zariski closure of $\mathcal{C}_{x_{1}}$ in $\mathbb{C P}^{n}$, then it has more than one holomorphic branch at $v$. Indeed, if the intersection of these ends is not empty, then from the fact that they are holomorphic and with the same slope, they should be equal. This is a contradiction with the assumption on $x_{1}$ and $x_{2}$. Hence, for $R$ sufficiently large, $V \backslash \mathscr{H}_{v, R}^{-}$is a fibration over $V_{v}$.

Proof of Theorem 1.1. Recall that the volume is always computed with respect to the induced measure of the ambient space. Using induction on the dimension $k$ of the variety, Proposition 5.1 and Theorem 4.1, there exists a rational number $q_{v}$ depending only on $v$ and on the variety $V_{v}$ such that the inequality

$$
\operatorname{vol}\left(\log \left(V \backslash \mathscr{H}_{v, R}^{-}\right)\right) \leq \pi^{2} q_{v} \operatorname{vol}\left(\mathscr{A}\left(V_{v}\right)\right)
$$

holds. There is a finite number of vertices of the logarithmic limit set, so there exists a positive real number $K$ such that the following inequality holds:

$$
\operatorname{vol}(\mathscr{A}(V)) \leq K+\sum_{v \in \operatorname{Vert}\left(\mathscr{L}^{\infty}(V)\right)} \pi^{2} q_{v} \operatorname{vol}\left(\mathscr{A}\left(V_{v}\right)\right)
$$

\section{REFERENCES}

[B-71] G. M. Bergman, The logarithmic limit-set of an algebraic variety, Trans. Amer. Math. Soc. 157 (1971), 459-469. MR0280489 (43:6209)

[BG-84] R. Bieri and J.R.J. Groves, The geometry of the set of characters induced by valuations, J. Reine Angew. Math. 347 (1984), 168-195. MR733052 (86c:14001)

[FPT-00] M. Forsberg, M. Passare and A. Tsikh, Laurent determinants and arrangements of hyperplane amoebas, Advances in Math. 151 (2000), 45-70. MR.1752241 (2001m:32060)

[GKZ-94] I. M. Gel'fand, M. M. Kapranov and A. V. Zelevinski, Discriminants, resultants and multidimensional determinants, Birkhäuser, Boston, 1994. MR1264417 (95e:14045)

[M1-02] G. Mikhalkin, Decomposition into pairs-of-pants for complex algebraic hypersurfaces, Topology 43 (2004), 1035-1065. MR2079993 (2005i:14055)

[M2-04] G. Mikhalkin, Enumerative tropical algebraic geometry in $\mathbb{R}^{2}$, J. Amer. Math. Soc. 18 (2005), 313-377. MR2137980 (2006b:14097)

[M3-00] G. Mikhalkin, Real algebraic curves, moment map and amoebas, Ann. of Math. (2) 151 (2000), 309-326. MR1745011 (2001c:14083)

[MR-00] G. Mikhalkin and H. Rullgård, Amoebas of maximal area, Int. Math. Res. Notices 9 (2001), 441-451. MR1829380(2002b:14079)

[NP-10] M. Nisse and M. Passare, (Co)Amoebas of linear spaces, arXiv:1205.2808.

[NS-11] M. Nisse and F. Sottile, The phase limit set of a variety, Algebra and Number Theory, to appear.

[PR-04] M. Passare and H. Rullgård, Amoebas, Monge-Ampère measures, and triangulations of the Newton polytope, Duke Math. J. 121 (2004), 481-507. MR2040284(2005a:32005) 
[PS-04] L. Pachter and B. Sturmfels, Algebraic Statistics for Computational Biology, Cambridge University Press, 2004. MR2205865 (2006i:92002)

[RST-05] J. Richter-Gebert, B. Sturmfels and T. Theobald, First steps in tropical geometry, Idempotent mathematics and mathematical physics, Contemp. Math., 377, Amer. Math. Soc., Providence, RI, 2005, 289-317. MR2149011(2006d:14073)

[R-01] H. Rullgård, Polynomial amoebas and convexity, Research Reports in Mathematics, Number 8, 2001, Department of Mathematics, Stockholm University.

NWF I-Mathematik, Universität Regensburg, 93040 Regensburg, Germany

E-mail address: Farid.Madani@mathematik.uni

Department of Mathematics, Texas A\&M University, College Station, Texas 778433368

E-mail address: nisse@math.tamu.edu 